\title{
LXIX. Absorption spectra and the solvate theory of solution
}

\section{Harry C. Jones}

To cite this article: Harry C. Jones (1912) LXIX. Absorption spectra and the solvate theory of solution, Philosophical Magazine Series 6, 23:137, 730-742, DOI: $\underline{10.1080 / 14786440508637269}$

To link to this article: http://dx.doi.org/10.1080/14786440508637269

曲 Published online: 08 Jun 2010.

Submit your article to this journal $\pi$

Џ Article views: 2

Q View related articles $\square$

Citing articles: 2 View citing articles 5 


\section{I.XIX. Alsorption Spectra and the Solvate Theory of Solution. By Harry C. Jones*}

[These investipations on the Absorption Spectra of Solutions were carried ont with the aid of Grants from the Carnegie Institution of W'ashington.]

DOZEN $†$ or more independent lines of evidence have A been discovered, all pointing to the conclusion that dissolved substances combine with more or less of the solvent. In a word, in dealing with solutions we must take into account not only the dissociation of the dissolved substance, but also its solvation, or power to combine with the solvent.

With this idea in mind it seemed highly desirable to study the absorption spectra of solutions. The absorption of light by dissolved substances is a resonance phenomenon; those wave-lengths that are absorbed finding something in the solution which they can throw into resonance with themselres. If the dissolved substance combines with more or less of the solvent, it seems reasonable to suppose that the substance would show different resonance from that which it would manifest in the uncombined condition. This conclusion is independent of whether the absorption of light by a dissolved subtances is due to molecules, ions, or electrons.

It seemed that some knowledge might be gained in individual cases as to whether there was solvation, and if so, what was its order of magnitude, by studying the power of solutions to absorb light under changing conditions of dilution, temperature, \&c.

This was, in general, the thought that led us to tilke up, six rears ago, the problem of the absorption spectra of solutions in as broad a way as possible; and the work has been in progress continuously since that time, under Grants generously awarded by the Carnegie Institution of Washington. We have had at our disposal in this work Rowland concave gratings ruled by Dr. Anderson, which gave us the large dispersion necessary to separate properly the absorption lines and bands.

The absorption spectra of somewhat more than 6000 solutions have been photographed, and some conclusions reached whose bearing on the solvate theory are of interest and we hope of some importance.

For details concerning the methods of work and the

* Communicated br the Author.

+ Zeitschr. phys. Chem. Ixxir, p. 32) (1010); Amer. Chem. Journ. xli. p. $56(1909 \%$ 
apparatus used, reference must be made to the earlier publications * of some of the results of this work. It should be stated that the grating spectroscope alone has been used.

The work begun by Jones and Uhlert had to do with three problems. They studied the effect on absorption spectra : of changing the concentration of the solution, of the addition of a deliydrating agent in the form of a second salt, and of the addition of water to non-aqueous solutions of the salt in question.

The results of this work were all interpretable in terms of the solvate theory of solution, and in terms of no other generalization; but it could not be said that any divect evidence for the theory was furnished by this earlier work.

The investigation which followed, however, furnished very direct evidence for the solvate theory. Jones and Anderson $\ddagger$ studied the absorption spectra of about 1200 solutions; including salts of cobalt, nickel, copper, iron, chromium, neodymium, praseodymium, and erbium in water and the alcohols as solvents. They varied in some cases the total number of ions in the path of the beam of light; in other cases the number of ions was kept constant and the number of molecules in the path of the light was varied. This was all accomplished by changing the concentration of the solutions and suitably changing the depth of the solution through which the light passed. By keeping everything constant except some one of these factors, and observing the effect on the various lines and bands when a given one of these quantities was varied, it was possible to determine what produced a number of the lines and bands.

Thus, the ultra-riolet absorption of cobalt salts was shown to be due to undissociated molecules or something contained within them, while the green cobalt hand was produced by the cobalt atom or something contained within it.

The absorption spectra of solutions of nickel salts were due chiefly to the nickel atom ; and, similarly, the absorption of copper salts in the red end of the spectrum.

The $\lambda 3300$ band of cobalt disappeared quickly with increase in dilution, even when the total number of molecules in the path of the beam of light was kept constant. It became more intense with rise in temperature. This band, obviously, could not be produced by ions molecules, or atoms,

* Carnegie Institution of Waslington, Publications nos. $(0,110,130$, and 160 . Amer. Chem. Journ. xxxvii. p. $1: 26$ (1007); xli. pp. 163, 276 (1909): xliii pp $37,87(1910)$.

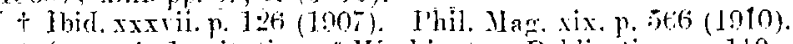

tanegie 1nstilution of Washingt nu. Publicalion mo. 110. 
but might be produced by simple hydrates formed by the breaking down of more complex hydrates with rise in temperature.

Cobalt salts show marked absorption in the red, only when very concentrated, at elevated temperatures, or in the presence of a dehydrating agent. The absence of red absorption at moderate concentration shows that this absorption is not due to ions. The conditions for the absorption in the red suggest that it might be due to molecular aggregates. This, however, is debarred by the fact that the red absorption increases with rise in temperature, while this would break down any such aggregates. We would, then, most naturally conclude that the absorption in the red was due to simple hydrates, since the conditions favouring this absorption favour the existence of such hydrates.

In a similar manner it was shown that the ultra-violet absorption of copper salts was connected with the solvation of the molecules-the absorption being smaller the more complex the solvate.

lirect spectroscopic enidence for the existence of solvates was furnished by Jones and Anderson in their study of salts of neodymium. A salt like neodymium chloride was found to have a very different absorption spectrum in water from what it had in methyl alcohol. Neodymium chloride in the proper mixture of water and methyl alcohol shows both the "water" bands and the "alcohol" bands. By varying the amounts of the solvents relative to one another, the relative intensities of the two sets of bands can be changed at will.

Neodymium nitrate in a mixture of water and methyl alcohol shows bands of the same character in the two solvents as the chloride.

Praseodymium chloride in a mixture of water and methyl alcohol shows the same kind of change as neodymium chloride. In this case an entirely new band appears in the alcohol, which has no analogue in the aqueous solution.

The existence of "water" bands and "alcohol" bands in the aqueous and alcoholic solutions, respectively, is interpreted by Jones and Anderson as direct evidence for the existence of hydrates in the aqueous solutions, and alcoholates in the alcohol solutions-in a word, is direct spectroscopic evidence for the solvate theory.

The largest amount and most direct spectroscopic evidence for the solvate theory of solution has been brought to light by the work of Jones and Strong, which has been in progress continuously during the past three years, and the results are 
as yet largely unpublished in any scientific journal *. A car eful study was made of the absorption spectra of cobalt salts in different solvents as affected by temperature. Glycerol solutions of these salts showod a different spectrum from aqueous solutions, indicating the presence of glycerolates in such solutions, as we have hydrates in aqueous solutions.

The effect of rise in temperature is to increase enormously the absorption of concentrated aqueous solutions of cobalt chloride, especially in the red. It is well known that such solutions turn blue on heating. The more concentrated the solution the lower the temperature at which this colour change takes place. There is a large amount of evidence which points to the conclusion that the change in absorption is due to a breaking down of the more complex hydrates with rise in temperature. If this is the true explanation of the phenomenon, the presence of a dehydrating. agent such as calcium chloride or aluminium chloride ought to cause this change in absorption to take place at a lower teinperature, since the dehydrating agent would assist the rise in temperature in breaking down the complex hydrates.

The results here are in accord with prediction. The addition of either of the above chlorides causes the great at)sorption in the red to take place in more dilute solutions at the same temperature, or in a given solution at a lower temperature.

It was further found that the temperature at which this marked change in the red absorption takes place is higher for aqueous and glycerol solutions than for solutions in other solvents ; showing that the "hydrates" and "glycerolates" are more stable with respect to temperature than other solvates.

The effect of rise in temperature on salts of chromium was also studied, and was shown to be similar to the effect of rise in temperature on cobalt salts-the higher the temperaturo the greater the absorption.

The action of dehydrating auents on chromium salts was similar to that on salts of cobalt.

\section{Absorption Spectra of Uranium Salts.}

A fairly comprehensive study was made of the absorption spectra of uranium salts, to see especially whether there was evidence obtainable for the existence of definite solvate bands in different solvents. We studied both uranyl and uranous compounds.

* See Caraegie Institution of Wasbington, Publications 130 and 160. 
It was soon found that an aqueous solution of uranyl chloride shows absorption bands in different positions from those of solutions in methyl alcohol. These differences were sufficiently great to merit careful study.

What these differences are can be best seen by giving tho wave-lengths of a few bands in the different solvents.

\section{Uranyl Chloride.}

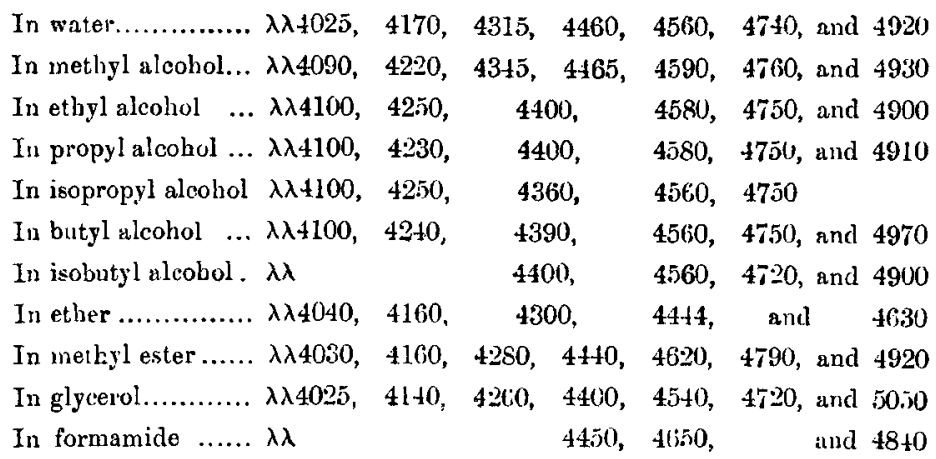

The absorption spectra of uranyl nitrate in mixtures of water and methyl alcohol were photographed. The absorption in pure methyl alcohol was much greater than in pure water. As water was added to the alcoholic solution the absorption became less and less. In the mixed solvents the bands became very broad. A careful study of the structure of these broad bands showed that they were the "water" and "alcohol" bands coexisting; and not one set of bands shifted in position, and this, as we shall see, is an important point.

A simple method of reducing uranyl to uranous salts in solution was found to be the action of nascent lydrogen. This was obtained by introducing into the aqueous solution of the uranyl salt a bar of zinc, and then adding the acid of the salt in question. In this way uranous salts could be prepared, and several of them were found to have sufficient stability for our purpose. Uranous chloride, uranons bromide, and uranous acetate were thus made and their absorption spectra photographed and studied. These salts proved to be very uselul for the detection of "solvent" bands, or the effect of the solvent on the absorption spectra of salts dissolved in it. The uranous bands are numerous, and what is far more important for our work, they are well defined with comparatively sharp edges, and their wave-lengths can be accurately determined. In this respect they are second only to certain lines of 
neodymium. Some of the most conclusive evidence for solvation iu solution, furnished by our spectroscopic work, was with the uranous salts.

The spectrum of uranous chloride in water is quite different from the spectrum in methyl alcohol, and this, in turn, very different from the spectrum in acetone. A glance at the plate for these solutions would lead us to conclude that we were dealing with three very different absorption spectra.

In a mixture of water and methyl alcohol, as the amount, of water increases the water hand $\lambda 6750$ comes out, gradually increasing in intensity. The methyl alcohol band $\lambda 5050$ to $\lambda 4850$ which is probably double, narrows on the red side into a band at $\lambda 4850$. Methyl alcohol bands $\lambda 4770$ and $\lambda 4600$ practically disappear and $\lambda \pm 670$ becomes very weak. In their places appear the water bands $\lambda 4700$ and $\lambda 4550$. The methyl alcohol band, $\lambda 4300$ to $\lambda 4450$, becomes weaker and breaks up into a band at $\lambda 4400$ and a band at $\lambda 4280$. The methyl alcohol bands $\lambda 4230$ and $\lambda 4120$ apparently come together, as the amount of water present relative to methyl alcohol increases, and become the water band at $\lambda 4160$.

It is thus obvious that the aqueous solution shows very different absorption bands from the solution of uranous chloride in methyl alcohol.

The addition of ethyl alcohol to an aqueous solution of uranous chloride produces a marked change in the spectrum, the ethyl alcohol bands being very different from the water bands. As the amount of ethyl alcohol present relative to water increases, the ethyl alcohol bands come out and the water bunds gradually disappear.

The addition of acetone to a methyl alcohol solution of uranous chloride brings out a number of "acetone" bands between $\lambda 6000$ and $\lambda 6500$. A strong absorption band also appears from $\lambda 6500$ to $\lambda 6800$. There is also an acetone band at $\lambda 5600$.

The addition of acetone to an aqueous solution of uranous chloride produces a marked change in the spectrum, the "acetone" bands being very different from the "water" bands. The acetone solution absorbs much less in the region $\lambda 6500$. The aqueous solution has a characteristic band at $\lambda 6750$. There are acetone bands at $\lambda 4920, \lambda 4750$, and $\lambda 4590$; and water bands at $\lambda 4980, \lambda 4700$, and $\lambda 4570$. The absorption of uranous chloride in ethyl alcohol is very similar to the absorption in methyl alcohol, as would be expected; the methyl alcohol bands being of slightly shorter wavelengths.

The absorption spectrum of uranous chloride in glycerol 
was photographed, also when water was added to the glycerol solution.

The absorption spectrum in glycerol was found to be very different from what it was in water.

The absorption spectrum of uranous chloride in a mixture of methyl alcohol and ether was photographed. The methyl alcohol solution showed complete ultra-violet absorption 10 wave-length $\lambda 3700$. The addition of ether increased the absorption, the absorption of the short wave-lengths extending now to $\lambda 3800$.

In methyl alcohol there was considerable general absorption in the region $\lambda 4300$. With the addition of ether the absorption was almost complete from $\lambda 4100$ to $\lambda 4450$. The addition of ether caused the uranyl bands to shift slightly towards the red without, however, changing their generai charncter. The magnitude of this shift can be seen from the following table.

Uranyl chloride bands in methyl alcohol.

$\lambda \lambda 3880,4000, \quad 4110, \quad 4240,4400,4610,4780.4930, \quad 5050$,

Cranyl chloride in methyl alcohol to which ether is added. $\lambda \lambda 3890,4010,4140,4260,4440,4630,4790,4960,5050$.

The effect of the presence of ether is thus very pronounced, not only causing the bands to shift slightly towards the red, but also increasing the amount of absorption.

It should be stated that in the work with the uranous salts, there were present in the solution in addition to the salt a little of the free acid of the salt and a small amount of the zinc salt. While these probably had a slight effect on the absorption spectra, the effect must have been far too suall to have produced such changes as those recorded above for the different solvents.

Having found such abundant exidence for the effect of the solvent on the power of the dissolved substance to absorb light, in the case of the few solvents referred to above, it seemed very desirable to bring other solvents $w$ ithin the scope of this work, and this has been done. Since the neodymium lines and bands are very sharp, it was selected as tbe principal substance to be used in studying the effect of the solvent on the absorption spectra of the dissolved substance, where a large number of solvents was to be employed. This phase of the work has been very recently carried out by Jones and Strong. 
Absorption Spectra of Neodymium Salts.

The following nomenclature will be used in describing the neodymium absorption spectra.

$\alpha$ group in the region $\lambda 3400$ to $\lambda 3600$.

$B$, at about $\lambda 4300$.

$\gamma \quad, \quad$ from $\lambda 4600$ to $\lambda 4800$.

$\delta$, from $\lambda 5000$ to $\lambda 5400$.

$\epsilon \quad, \quad$ in the region $\lambda 5800$.

$\zeta$, at $\lambda 6300$.

In designating the neodymium spectra we start from the violet end of the spectrum. This is the natural method when a grating is used. It is doubtful whether, in the near future, the ultra-violet spectrum of neodymium can be studied much farther than we have done, so that this is the natural end of the spectrum at which to begin. It is, on the other hand, probable that there are many neodymium bands farther down in the infra-red than we have gone; and when these have been worked out they can then be named in the natural order.

'The change in the absorption spectrum of neodyminm chloride as the solvent is changed can be seen best by expressing the results in the following form. The abbreviations nsed are: "d" diffuse, "fa" faint, "fi " fine, " $h$ " hazy, " $i$ " intense, " $n$ " narrow, " sh" sharp, "st" strong, "we" weak, "wi" wide.

The following results (pp. 738-9) obtained with neodymium chloride show the effect of the solvent on the absorption spectra of solutions of this compound. The bands of the different solvents bave different wave-lengths and different relative intensities.

Having found that the solvent played an important part in determining the absorption of light by the dissolved substances, Jones and Strong used isomeric organic solvents to see whether such closely related compounds would affect differently the power of substances dissolved in them to absorb light. They prepared solutions of neodynitum chloride in propyl and isopropyl alcohols, and in butyl and isobutyl alcohols, and photographed the absorption spectra of this salt in these isomeric solvents. The following results show different absorption lines and bands in the isomeric solvents.

Phil. Mag. S. 6. Vol. 23. No. 137. May 1912. 3 C 
738 Prof. H. C. Jones on Absorption Spectra
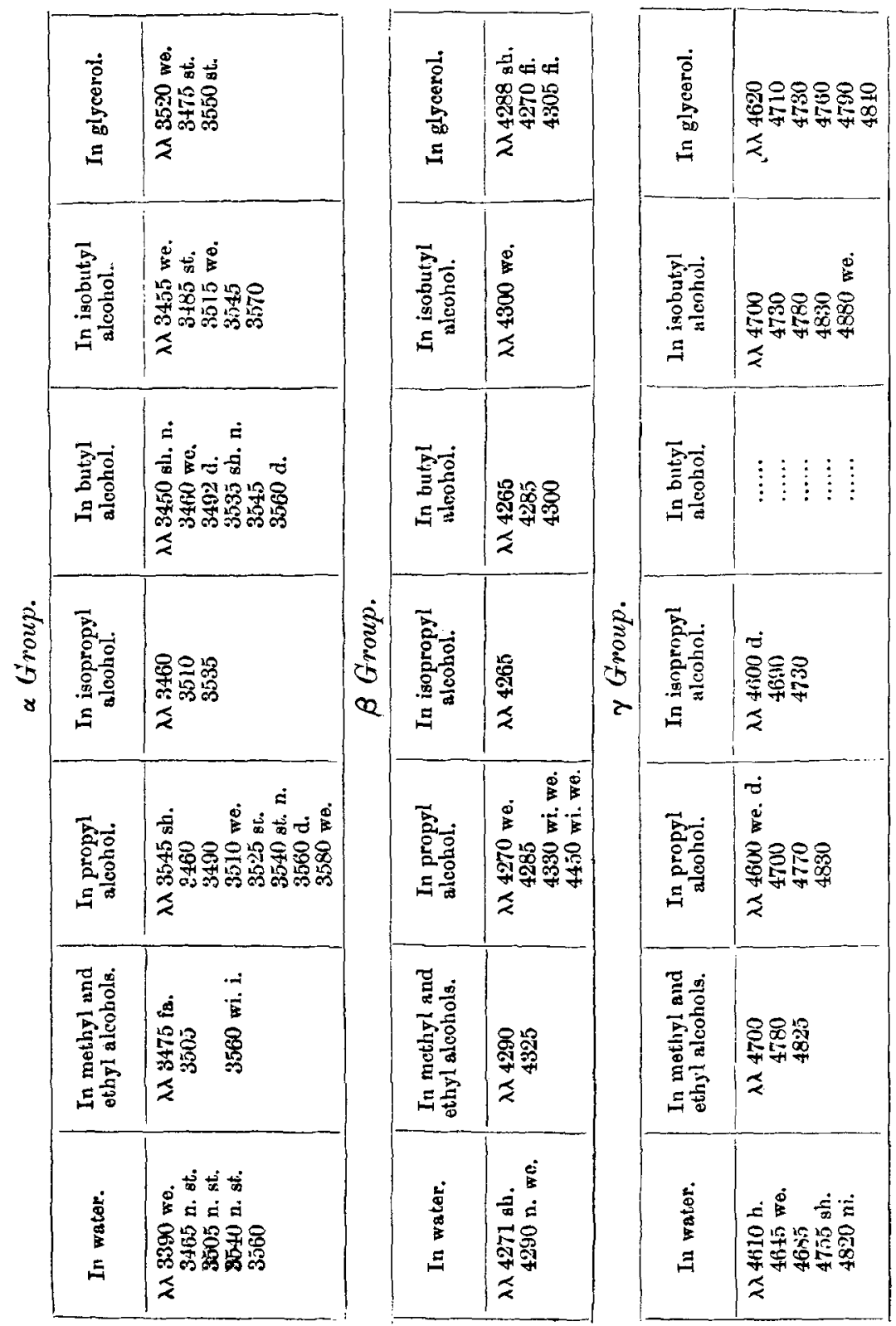
and the Solnate Theory of Solution.
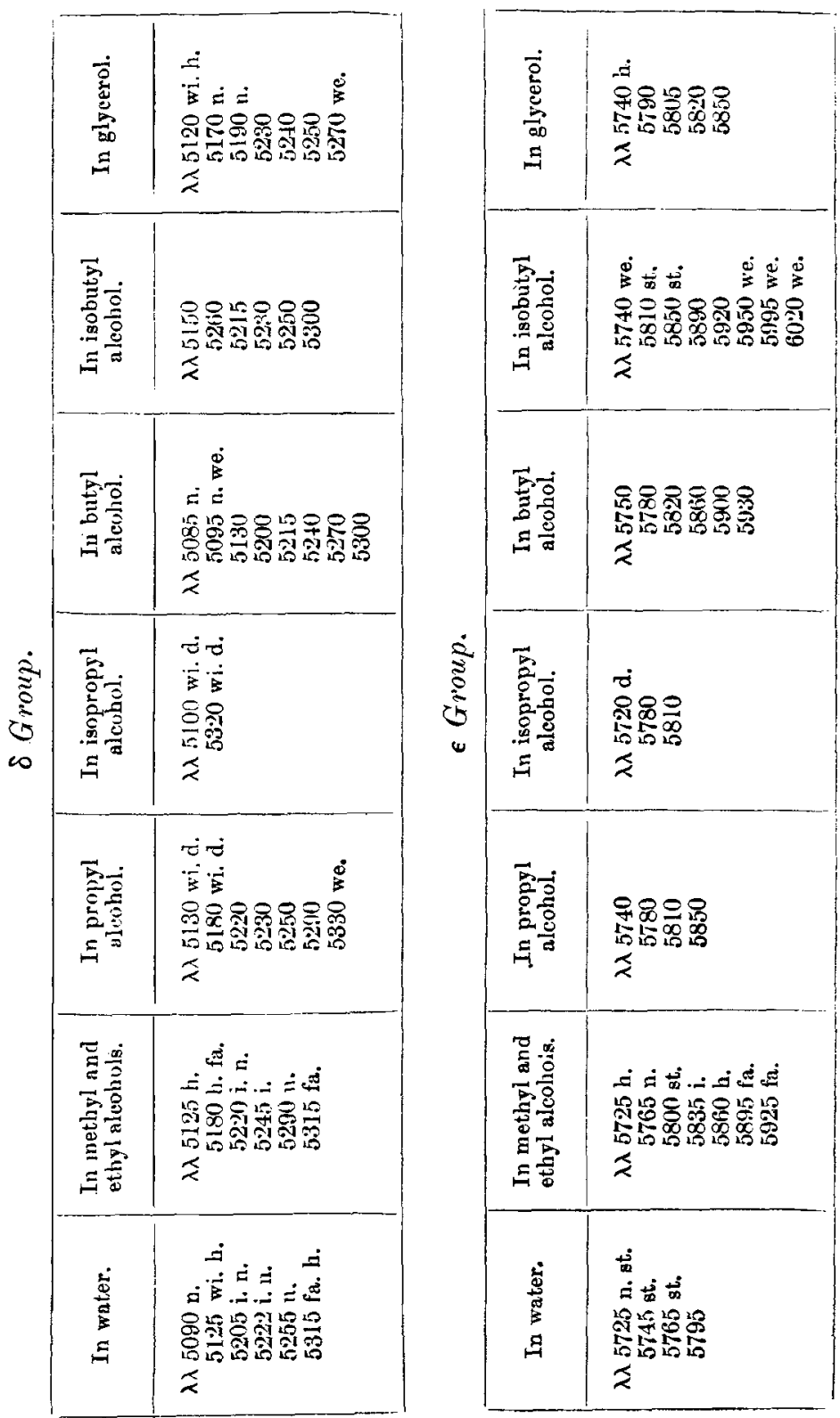
If we compare carefully the spectra of neodymium chloride in butyl and isobutyl alcohols, we find that the bands are weak and diffuse in isobutyl alcohol, and have different relative intensities from what they have in the butyl alcohol. The bands in butyl alcohol are very much finer and sharper than they are in isobutyl alcohol. Further, the bands of neodymium chloride in isobutyl alcohol have slightly greater wave-lengths than in butyl alcohol.

To eliminate the possibility of the effect of the solvent on absorption spectra being due to anything inherent in the nature of neodymium chloride, the nitrate of neodyınium was studied in the same way as the chloride.

The absorption spectra of neodymium nitrate in water, in methyl alcohol, in ethyl alcohol, in mixtures of these alcohols and water, in propyl and isopropyl alcohols, in butyl and isobutyl alcohols, in acetone and in mixtures of acetone and water, in ethyl ester and in formanide, were carefully photographed and studied. Results will be given in the case of neodymium nitrate only for the $a$ bands.

\section{a Bands.}

In water. Practically the same as the bands of neodymium chloride, but the bands of the nitrate are broader and hazier than those of the chloride.

In methyl and ethyl alcohol. There are only two bands in the a group, $\lambda 3465$ and $\lambda 3545$.

In propyl alcohol. $\lambda \lambda 3455,3500$, and 3585 .

In isopropyl alcohol. $\lambda \lambda 3460,3505$, and 3535 .

In butyl alcohol. $\lambda \lambda 3450,3500$, and 3540 .

In isobutyl alcohol. Ultra-violet absorption was so great that on the plate taken the a group did not appear. The absorption in general is the same as that of the chloride in this alcohol.

In acetone. $\lambda \lambda 3475$ and 3555 .

In ethyl ester. $\quad \lambda \lambda 3455,3500$, and 3540 .

The other groups of absorption bands of neodymium nitrate in the different solvents show differences in the wave-lengths comparable with the above; bnt these results suffice to show the effect of the solvent on the power of neodymium nitrate to absorb light.

The above evidence that the solvent plays an important part in the absorption of light by substances dissolved in it is strong. When we take into account the number of salts studied and the number of solvents employed, the evidence 
is little short of proof. The only reasonable question is how are we to interpret these facts? Before attempting to answer this question we should take into account also the following fact. We have seen that a salt dissolved in a given solvent is characterized by a definite absorption spectrum. When a salt is dissolved in mixtures of varying proportions of two solvents only two definite absorption spectra appear, one being characteristic of each solvent. One spectrum does not gradually change into the other as the composition of the mixed solvent changes, but only the relative intensities of the two spectra vary. Starting with that mixture of the two solvents in which both of the spectra are equally intense, if we diminish the amount of $a$ relative te $b$, the spectrum corresponding to $a$ becomes feebler and feebler, and the spectrum corresponding to $b$ more and more intense. This fact was first noted by Jones and Anderson, and since repeatedly confirmed by the work of Jones and Strong. We found that when neodymium chloride is dissolved in a mixture of methyl alcohol and water, it showed a definite set of "water" bands and a definite set of "methyl alcohol" bands. As the amount of water in the solution was decreased relative to the alcohol, the "water" bands decreased in intensity butremained in the same position. As the amount of alcohol in the solution was decreased relative to the water the "alcohol" bands decreased in intensity, but their position remained unchanged.

Jones and Anderson interpreted these facts as strong evidence in favour of the view that there are definite hydrates and definite alcoholates in the solution.

The spectroscopic evidence for solvation in solution furnished by Jones and Anderson has, as has already been stated, been increased many fold by the work of Jones and Strong. A large number of solvents and a fairly large number of salts have been used, and the existence of solvent bands in general established.

The question of the relative stability of the different solvates with respect to various physical and chemical agents has been studied at length by Jones and Strong by means of absorption lines and bands. It would lead us beyond the scope of this paper to discuss these results in detail. Suffice it to say that the hydrates in general are the most persistent of all the solvates, although this depends upon the conditions to which the solution is subjected.

Taking all of the spectroscopic work into account, I regard the evidence from this source as strongly supporting the solvate theory of solution as advanced in this laboratory more 
than a dozen years ago. The bearing of this theory upon Chemistry and other branches of Natural Science has already been pointed out ${ }^{*}$. Suffice it to say here that the solvate theory of solution is not at all at variance with the theory of electrolytic dissociation, but beantifully supplements it.

Johns Hopkins University, 1911.

LXX. The Unilateral Conductivity of Sulphated Accumulator Plates. By Robert G. Lunnon, B.S. $†$

THE high electrical resistance of accumulator plates 1 which have been left unused for a considerable time is well known, and is due to the presence of $\mathbf{a}$ film of a sulphate of lead on the negative plate. This resistance becomes normal on the application of a bigh potential for a few minutes, and the experiments to be described were carried out to investigate this change. This object was attained, and it was further found that the layer of sulphate exhibits the property of aluminium anode-films in having a resistance dependent upon the direction of the current.

The accumulators used were of the simplest Planté type, as used in large numbers for obtaining a high potential. A different plate was necessarily used for each experiment, but the properties of the cells in one tray were found to be sufficiently identical to make this of no consequence. The connexion between the rate of decrease of the resistance and the applied potential difference was examined by including

Fig. 1.

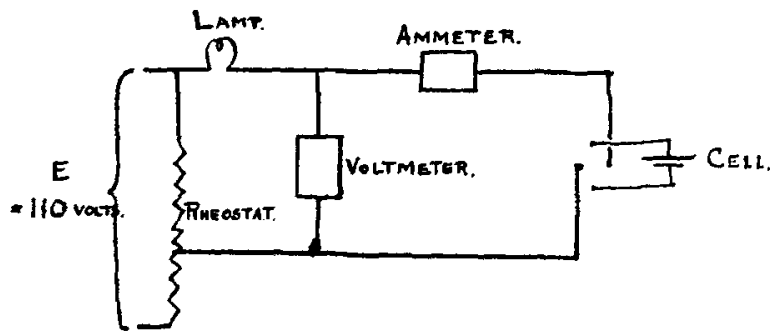

the cell in a circuit, as shown in fig. 1 . With this arrangement, any desired p.d. could be initially applied to the cell, and its diminution with time, together with the current going

* Zeitschr. phys. Chem. lxxir. p. 377 (1910). Journ. d. Chim. phys. ix. p. 217 (1911). Amer. Chem. Journ. xlv. p. 146 (1911).

+ Communicated by Prof, F. T. 'Trouton, F.R.S. 